On some points in the design of optical instruments

This content has been downloaded from IOPscience. Please scroll down to see the full text. 1902 Trans. Opt. Soc. 411

(http://iopscience.iop.org/1475-4878/4/1/303)

View the table of contents for this issue, or go to the journal homepage for more

Download details:

IP Address: 132.239.1.230

This content was downloaded on 07/09/2015 at $16: 10$

Please note that terms and conditions apply. 


\section{ON SOME POINTS IN THE DESIGN OF OPTICAL}

\section{INSTRUMENTS.}

Paper by C. V. Drysdale, D.SC., Member of Council.

Reud before the Optrcal Society on December 18th, 1908.

$\mathrm{O}^{\mathrm{N}}$ being again invited to read a paper before a meeting of select, and I finally adopted the above title not without considerable thought and many misgivings. When I read my paper on the Testing of Optical Instruments before you in the early part of this year, the subject was one of general interest, in that the retail Opticians were concerned in being able to ascertain the optical quality of the instruments coming into their hands. When, however, we come to consider the production of these instruments, we are met with the disagreeable fact that although this country is a large consumer of optical apparatus it is by no means a large producer, and further that as the demand for cheaper and better worls has increased, our position as manufacturers has become relatively worse. It is, therefore, of the greatest importance that we should examine into this state of affairs and see how they may be improved, and it is greatly to be hoped that both manufacturers and retail Opticians alike will co-operate in doing all in their power to bring about this improvement.

Two great factors enter into the successful manufacturing of optical instruments as with other commodities. One of these is the manufacturing methods adopted, the other is the design of the optical and mechanical details. It is no use being able to turn out mechanical work very thoroughly indeed if you cannot use the proper lenses in order to produce images which are very perfect in definition; and $I$ am afraid that is a subject which has very sadly been left alone in this country. 
We stand, practically, as far as the theory of technical optics is concerned, where we did 70 years ago. In both of these ways we have been seriously falling behind of recent years, but in the former we are now keenly alive to the matter, and there is no doubt that with improved machinery and workshop processes. much lost ground may be regained. (Hear, hear.) In the mechanical design of our optical instruments, too, we are not much behindhand, in fact, in certain respects English mechanical design is probably superior to all others. When, however, we turn to the optical performance of our instruments we are forced to admit that in almost all directions German instruments are in every way superior, and hardly a month passes without some improvement in telescopes, photographic lenses, or microscope

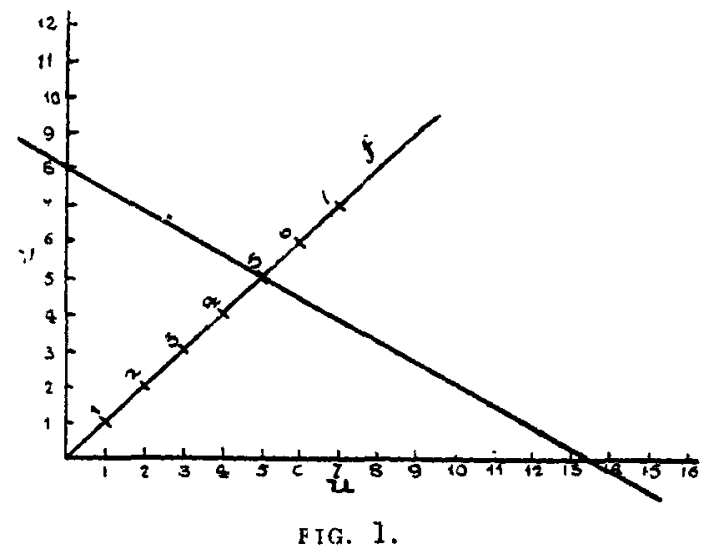

objectires emanating from the Contment, while but little advance is being made in England.

What is the reason of this? If we turn to the records of English work in the domain of techinical optical theory we find that scarcely any progress has been made since the appearance of Coddington's "Optics" in 1829, and the first achromatic comhination was produced by Dollond. Not one book of importance has been produced during this time until the translation of Professor Lummer's "Photographia Optics" was made by Professor S. P. Thompson in 1900. Not one of our English professors has made a special study of this subject. On the Continent we find that the principle of the correction of spherical 
aberration was first enunciated by Euler, that of the correction of coma by Frauenhofer, and extended by Abbé, that of the employment of the aplanatic points of a sphere by Amici, while the rules for eliminating the errors of astigmatism, curvature, and distortion, were respectively put forward by $\mathrm{Rudolph}$, Petzval, and Lummer. The conditions for flattening the field are indeed given in Coddington, but it remained for Abbé and Schott to make them practicable by the production of the anomalous Jena glasses. In addition we have the names of Steinheil, Von Seidel, Pulfrich, Czapski, Kaempfer, and many others who have distinguished themselves in this field. With such a catalogue of names and achievements it is little wonder that the science and

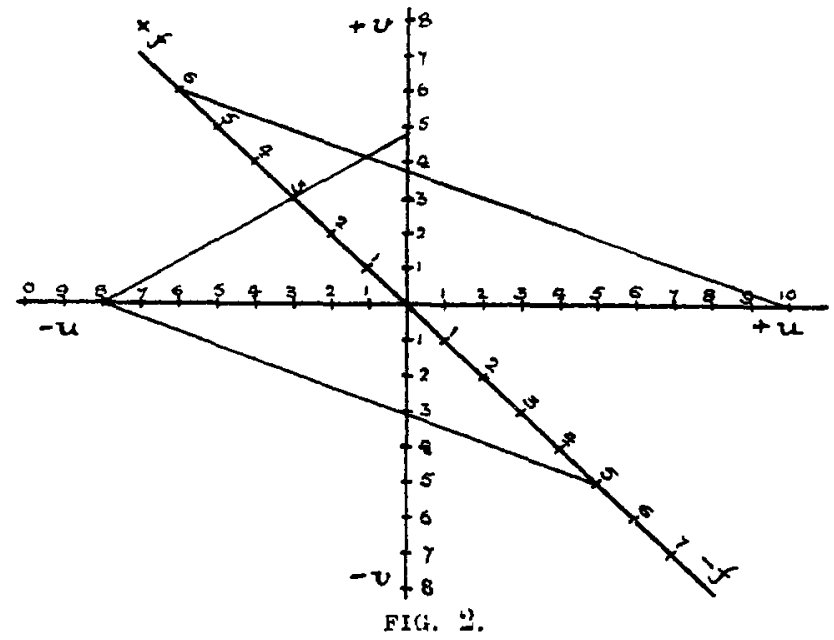

art of optics has made such strides on the Continent as almost to eclipse the work of British manufacturers, and it is only to be hoped that we may recognise as strongly as possible our backwardness in these directions, and that we may take steps to remedy it.

$\mathrm{My}$ apology, therefore, for bringing before you this evening a paper on such an intricate matter, must be the importance of the subject, and my reason for doing it threefold. In the first place I hope to be able to strip the subject of some of its intricacies, and to make the leading principles of design intelligible to those who have only an elementary knowledge of optics; in the 
second place, to indicate a few new methods of surmounting these difficulties, and thirdly, and most important, with the object of pleading with our few scientific manufacturers that they should come forward and enlighten us as to their methods, as their continental contemporaries have so freely done. And in most cases. they have done so not only for the benefit of their own country but for the benefit of the world. I do not mean to imply altogether that we should slavishly copy the German methods, but that we should seriously study the subject. Only by papers and discussions on this important subject ean we regain our place, and I hope that the spirit of co-operation will induce us to lay aside all petty trade secrets and jealousies for the common good.

I have emphasised this latter point for another reason. When

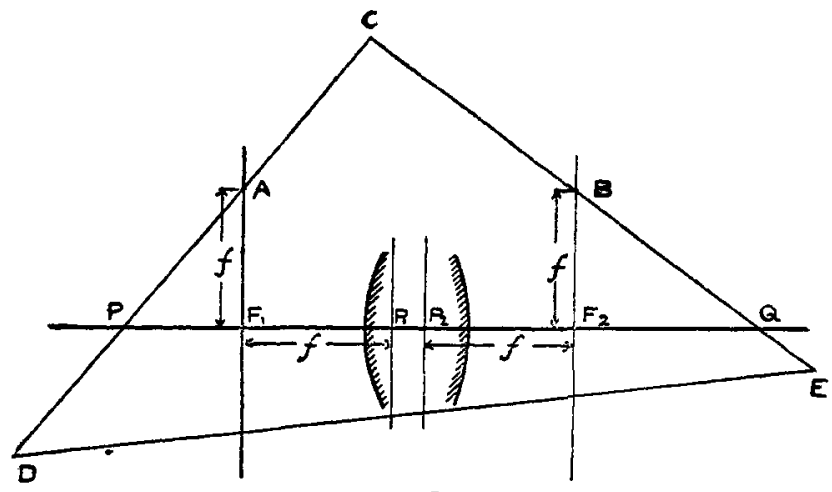

FIG. 3.

one comes to study the work of German scientific men in this or any other direction, one is immediately impressed with the immense amount of abstract mathematical or other scientific reasoning which there appears, and the colossal amount of what can only be described as intellectual drudgery they seem willing to undertake. In no subject is this more strongly marked than in that of technical optics, and I fear that if advance in this direction is only to be obtained by slavishly following the mathematical and numerical computations adopted by the Germans, we are not likely to rapidly catch up. Only those who have attempted it can realise the labour involved in the designing of such an instrument as a photographic lens, the hours and days of pure numerical computation required, which may have in 
the end to be considerably modified by changes in the quality of the glass, or a slip in the calculation. No wonder, therefore, that English Opticians have very commonly an extreme contempt for theoretical design, and have preferred to obtain their results almost by pure experiment; and even at the present time there are a great many who, while admitting that the highest results can only be obtained by scientific methods, are yet doubtful as to the commercial practicability of adopting them. It is characteristic of the Briton that he prefers to get his results by direct short cuts, while his German cousin uses steady perseverance; and while we study and pay the greatest respect to the grand optical work of the Germans, there is no harm in trying if we can to inf use some British methods into them. For my own part, I feel certain that it can be done with great advantage.

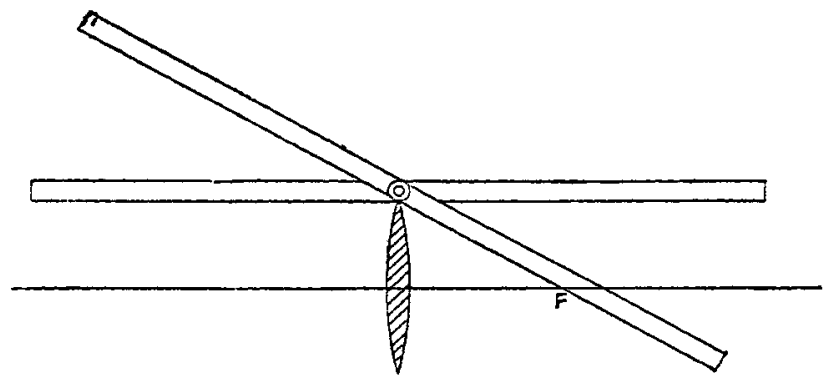

FIG. 4 .

In designing an optical system for any purpose there are two main stages. The first is to arrange our lenses, prisms, etc., so as to get a system of a certain focal length, giving an image of the proper magnification, and sufficient illumination and field of view. The second stage is to consider the errors of aberrations of such a system and to settle the curres and glasses so as to eliminate these errors.

\section{PART I.}

In settling the lenses, etc., of any optical system, it is, of course, necessary for us to know how they modify the course of the light passing through them. There are three ways of doing this, two of which may be distinguished as geometrical methods, while the third may be termed the curvature or physical method. 
In the first of these the course of the light is specified by tho positions of the conjugate foci, and the lateral distances of the intersections of conjugate rays with reflecting and refracting surfaces. This may be termed the ordinary or text-book method. The second is due to Von Seidel, who departed from the ordinary method in substituting the angles of inclination of conjugate rays to the axis for the distances. The third or curvature method is the legitimate extension of the system used by Opticians, and consists in following the alteration of the convergence of the light through the system. Of these the first is undoubtedly the worst, the second has led to the magnificent generalisations of Von Seidel, while the third is comparatively untried. In my opinion, however, we shall find that this method will do much to simplify all optical processes even in the calculation of aberrations, and I have ventured to illustrate this in the second section of this paper. In fact, it is most noticeable whenever lens formulæ are being dealt with how the results will persist in expressing themselves in curvature form. It is as if our mathematical symbols knew their optics better than we do, and keep pointing out how they should be treated.

I need say little here as to how the course of the light may be traced through any system by any of these means, as it is a fairly simple niatter. I have, however, one suggestion to make concern. ing nomenclature which I should like to bring before your notice.

It has been wisely decided to employ as our unit of curvature the diopter, that having a radius of one meter. Now in optical instrument work we frequently have to deal with large curvatures and small distances, the latter being more conveniently expressed in centimeters or millimeters. Why should we not therefore apply our ordinary metric prefixes to the diopter, and use a table like this?

RADIUS OF CURVATURE. CURVATURE.

1 kilometer .1 millidiopter $=1 / 1000$ th diopter

1 meter ..... 1 diopter

1 decimeter $\ldots 1$ dekadiopter $=10$ diopters.

1 centimeter . . 1 hectodiopter $=100$ diopters

1 millimeter. 1 kilodiopter $=1,000$ diopters

1 micron $\quad \ldots 1$ megadiopter $=1,000,000$ diopters.

When designing with distances and radii in millimeters the corresponding reciprocals would be the curvatures in kilodiopters. 
Graphical Methods.-The numerical calculation of the path of the light through a system is frequently tedious, and as to make it intelligible it is best always to draw the course of the rays; it is troublesome to have to change from the drawing board to calculation and vice versa $\hat{a}$ repeatedly. What would be most eminently desirable in the first stages of designing is some method by which the course of the rays could be indicated on a drawing without calculation, and I have recently given some little attention to this subject to see whether any convenient methods are available.

Sir Howard Grubb's Method.-Where the system to be examined is made up of any number of thin lenses, this method for obtaining the positions of the conjugate points is of considerable value, and an apparatus suitably made on this principle is of great use in lens designing. As is well known, Sir Howard Grubb's method consists in reading off the conjugate distances

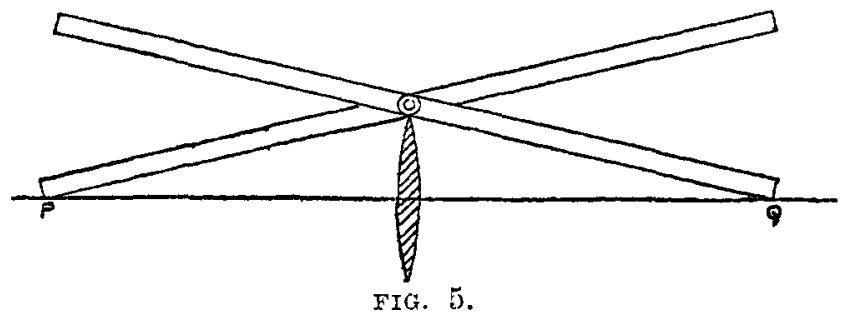

and the focal length on three scales, the scales for the two distances being set at right angles to one another, while the focal length is indicated on a scale at $45^{\circ}$ to the other two. If a straight edge is employed, which is pivotted about a point which is set to the proper distance on the scale of focal length, then the intersection of this straight edge with the two axes give two conjugate positions, as is indicated in fig. 1, and however the edge is moved these pairs of conjugate distances can always be read off immediately. So far $I$ have seen this arrangement described, it has been applied only to real foci produced by convergent lenses, but it is obviously extensible by producing all three scales as in fig. 2 , so as to be used for real or virtual foci produced by either convergent or divergent lenses. It is possible with such an apparatus in a few seconds, therefore, to find the position conjugate to any given position with a lens of given 
focal length, or to find the focal length required for any two conjugate distances.

I have, however, ventured to modify the method further, so that it may be employed by a draughtsman without reference to any separate apparatus, and to make it equally applicable to lenses of any thickness. All that is required for the purpose is a rule composed of two straight edges at right angles; in fact, for many purposes an ordinary set square will suffice. To make use of this method all that is necessary in finding the conjugate positions for any given lens is to measure off the focal length of the lens on both sides of it, as shown in fig. 3, and to then stick two pins, $A$ and $B$, in the drawing board in these two focal planes, but at a height from the axis equal to the focal length. If then the set square, CDE, is placed with its two edges against the points $A$ and $B$, the points where these edges intersect the axis will always be two conjugate points, and it is therefore possible on the drawing to see in a moment how the position of the image varies with the position of the object in any case. The great convenience of this method is that it is possible in addition to notice the effect of varying the position of the lens, or the separation between the different components of a system.

Although the method last described is exceedingly convenient and accurately gives the positions of the conjugate foci in accordance with the rules to a first approximation, it has the objection that it does not accurately indicate the course of the rays of light from the object to the image, and it can give no indication, therefore, of the conjugate positions for oblique pencils. I have, therefore, been led to devise two methods, one approximate, which is convenient for use with thin lenses; the other, which can be made as accurate as may be desired, and will give the course of the rays when refraction takes place at any refracting and reflecting surface. In the first of these all that is required is a jointed rule, preferably crossing like a pair of scissors, with two straight edges which can be fixed at any desired angle. In fig. 4 an application is shown of this rule to the finding of the position of an image formed by a simple convex lens of known focal length. In the case of a thin lens the refraction at any zone of it is, of course, equivalent to the case of refraction by a thin prism of definite angle, and we know that with a thin prism the deviation produced by it is to a first approximation indepen- 
dent of the direction of the incident light. Consequently, if, as in the figure, we have any rays striking the lens at any distance from the centre, it is only necessary for us to stick a pin in the paper where the ray cuts the principal plane of the lens, and to place the rule so that the intersection of the two straight edges touches the pin. If, now, one edge of the rule is held parallel to the axis of the lens, while the other is bent to pass through the focal point, it clearly represents the path of a ray parallel to the axis being converged to the focus, and consequently gives the deviation produced by the particular zone of the lens. Secondly, if the rule is clamped with its edges at this angle, and it is then rotated about the pin, the two edges will always represent conjugate rays, and if one edge is made coincident with the incident ray, the emergent ray will be given by the other edge and can be drawn on the paper, fig. 5. Fig. 6 shows the diagram obtained

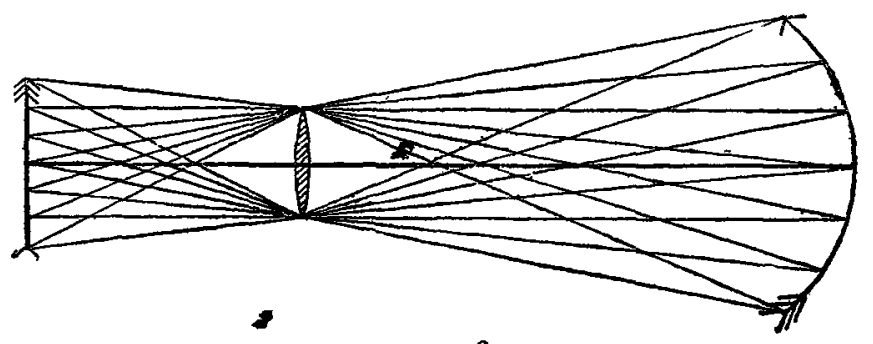

FIG. 6.

by applying such a rule to find the image produced by a straight line object at a distance from a convex lens, and indicates very strikingly the curvature of the image produced. Of course, it is well known that the curvature of the image surface is not definite, as we have three surfaces, one containing the primary focal lines, the other the secondary lines, and the third theircles of least confusion, but the directions of the rays of light are, given with sufficient approximation to be useful for design purposes, provided that the angle of deviation is not large.

It may also be pointed out with advantage that this method is equally suitable to enable one to find the directions of conjugate rays in a lens of any thickness, provided that the curves are not very deep, and that the equivalent focal length is known. In this case, all that need be done is to stick two pins in the 
paper instead of one, at the same height from the axes on the two principal planes of the lens, and if the rule is then held so that it touches both of these pins, the conjugate rays will be given as before.

\section{An Accurate Method of Graphically Determining the Directions of Conjugate Rays.}

Although the methods given above are of great utility in first scheming out a lens system, they can hardly be utilised when any more definite information concerning the lens system is required. It will be shown later that all the various errors to

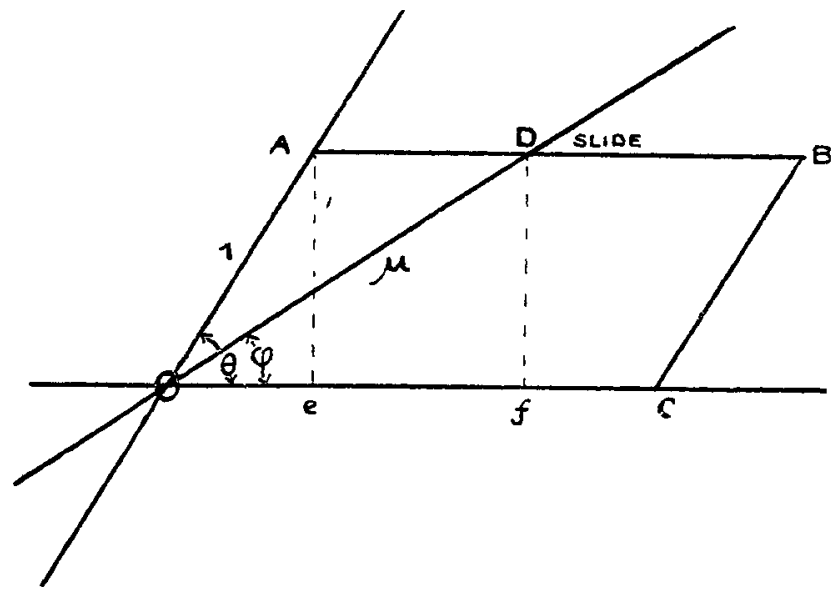

FIG. 7 .

which reference has already been made depend upon the direction of the light and the distance from the axis at which the ray cuts the refracting surface. In order, therefore, to get a complete representation of the aberrations of a refracting system, as has been done by Von Seidel, it is necessary to follow mathematically the course of the light from surface to surface through the system, finding the refraction and the aberrations at each surface. The complexity of this process is extremely great, and it is here especially that the writer feels that some means of sim plification ought to be attempted. If, therefore, it were possible accurately to cletermine the course of light through the lens 
system without calculation, it would be possible to calculate the aberrations with very much greater ease.

Our fundamental formula for refraction is well known and may be stated in these terms, that whenever light is refracted on passing from one medium to another, the product of the refractive index of the medium and the angle which the light makes with the normal to the surface remains constant (this product divided by the radius of the surface is commonly known as Abbé's optical invariant). Consequently, if we were able to make any form of instrument or rule in which this law is fulfilled we should be able to get the directions of conjugate rays to any required degree of accuracy, and the attainment of this is not difficult. In fig. 7, OABC represents a frame jointed at all

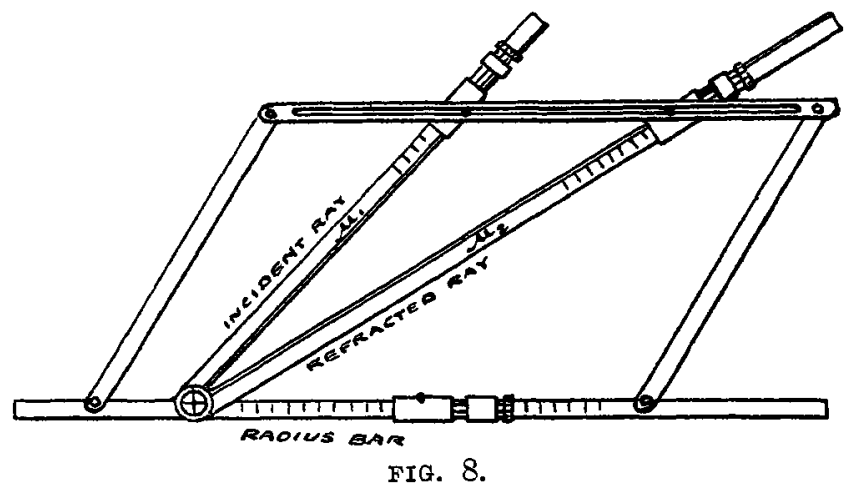

four angles, so that $A B$ is always parallel to $O C$. $O D$ represents another rod hinged at $O$ and having at the point $D$ a pin which slides along $A B$. The distance $O D$ is made $\mu$ times $O A$. Then if $O C$ is the normal to any refracting surface and $O A$ is set in the direction of any incident ray, OD will be the corresponding emergent ray. For if we consider the perpendicular distances Ae and $\mathrm{D} f$ we know that $\sin \theta=\frac{\mathrm{A} e}{\mathrm{OA}}$ and $\sin \gamma=\frac{\mathrm{D} f}{\mathrm{OD}} \therefore \frac{\sin \theta}{\sin \gamma}$ $=\frac{\mathrm{OD}}{\mathrm{OA}}$ since $\mathrm{A} c=\mathrm{D} f$, hence $\frac{\sin \theta}{\sin \gamma}=\mu$.

Fig. 8 represents a rule designed on these principles, applicable to finding the refraction at any surface between any two media. 
Two hinged bars are shown, each of which has a sliding point which can be set to any desired refractive index. Fig. 9 shows the application of the simpler form of instrument to find the conjugate rays in an actual case. Of course, if divided circles were attached, the angles of incidence and refraction, and of inclination to the axis, could be directly read off, to a considerable degree of accuracy. In my opinion such a rule, if well constructed, would give us the direction of the light with sufficiently great accuracy for it only to be necessary for us to calculate the aberrations at each refraction.

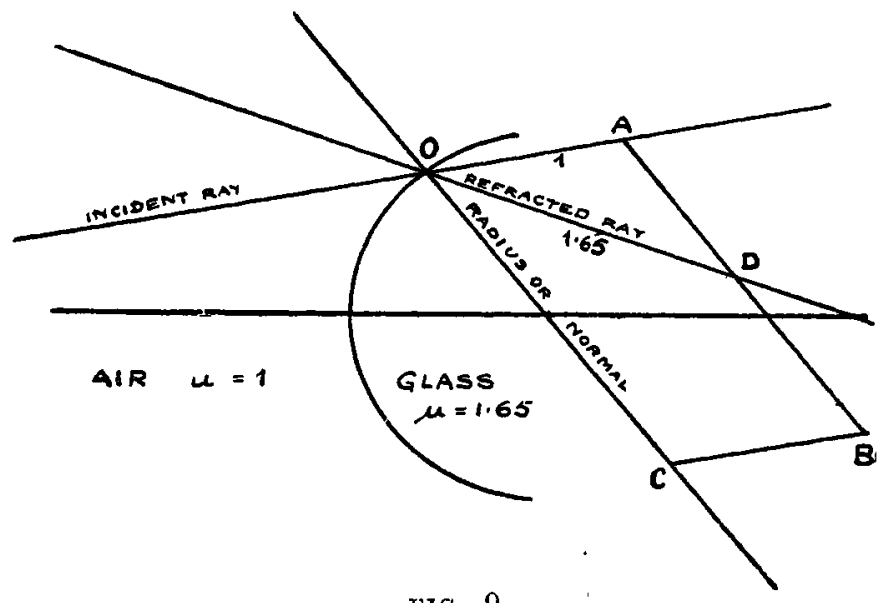

FIG. 9.

\section{Part II.-Aberrations and their Correction.}

We now come to the part of our subject which is of a more difficult nature, and can only be attacked completely by somewhat intricate mathematical processes. Nevertheless, the subject is full of interest, both from the scientific and commercial standpoint, and although it would be manifestly impossible to deal with it thoroughly in one or even several papers, I have ventured to write the following in the hope that it may interest some sufficiently to pursue it further, and that it may evoke scientific discussion.

In the first place, it will be well if we state scientifically what is meant by an aberration. If we make use of the ordinary approximate formulæ for lenses and mirrors, we find that to every point of the object corresponds a point in the image; 
further, that if the object lies in a plane perpendicular to the axis, the image lies in a similar plane, and finally, that the image is exactly similar to the object. When an image is formed from an object in this manner, the two are said to be in collinear relationship, and this relationship is satisfied by the approximate or Gauss formulæ. When, however, we come to examine actual lenses more closely, we find that the conditions for collinear relationship are by no means satisfied, in that the light emanating from a point in the object is not focused to a point in the image, that the best image of a plane object lies on a curred surface, and that there is a certain departure from exact similarity between image and object. The deviations of the actual paths of the light from those which they should follow according to the approximation formulæ of Gauss are their aberrations, and it is convenient to classify these aberrations according to the effect they produce on the image.

This classification may be given as follows:-

\section{Primary Aberrations :}

Chromatic aberration. $\quad\left\{\begin{array}{l}\text { Position. } \\ \text { Magnification. }\end{array}\right.$

Central spherical aberration.

Coma.

Radial astigmatism.

Curvature of field.

Distortion.

\section{Secondary Aberrations :}

Secondary chromatic aberration.

Chromatic differences of spherical aberration.

Chromatic differences of other aberrations.

We shall not refer to the secondary aberrations, but a little attention will be given to each of the primary aberrations and their correction.

Chromatic Aberration.-The general principles of this aberration can be found in many text books, but it is much more simply dealt with by the curvature or convergence method. Taking first the case of a simple thin lens, we have $\mathrm{F}=(\mu-1) \mathrm{C}$ where $\mathrm{C}$ is the total curvature of the lens and $\mathrm{F}$ its convergence. Consequently, if we consider light of any two different colours, we have $\mathrm{F}_{1}=\left(\mu_{1}-1\right) \mathrm{C}$ and $\mathrm{F}_{2}=\left(\mu_{2}-1\right) \mathrm{C}$ and subtracting $\mathrm{F}_{2}-$ $\mathrm{F}_{1}=\left(\mu_{2}-\mu_{1}\right) \mathrm{C}$ or $\Delta \mathrm{F}=\mathrm{C} \Delta \mu$ where $\triangle \mathrm{F}$ is the difference of the convergences of the lens for the two colours, and $\Delta \mu$ is the dispersion or difference between the refractive indices of the glass 
for those colours. The quantity $\triangle F$ I have ventured to call the chromatic convergence of the lens, and we therefore see that the chromatic convergence of a lens is equal to the product of its curvature and the dispersion of the glass.

$$
\begin{aligned}
& \text { But from the first equation, } \mathrm{C}=\frac{\mathrm{F}}{\mu-1} \text { and hence } \Delta \mathrm{F} \\
& =\mathrm{C} \Delta \mu=\frac{\mathrm{F}}{\mu-1} \Delta \mu=\frac{\mathrm{F}}{\frac{\mu-1}{\Delta \mu}}=\frac{\mathrm{F}}{\nu} \text { where } \nu=\frac{\mu-1}{\Delta \mu}
\end{aligned}
$$

and is called variously the "achromatic refractivity" or the "efficiency" of the glass for the two colours concerned. In the catalogues of optical glass, these efficiencies are given, and con. sequently the chromatic convergence of any lens is given by simply dividing its convergence by the efficiency of the glass of which it is composed.

Nothing is easier when this result has been obtained than to find the chromatic convergence of any number of thin lenses in contact and thus to calculate an achromatic combination. For two lenses having convergences of $F_{2}$ and $F_{2}$ in contact, we have $F_{1}+F_{2}=$ $F$ the combined convergence, and $\frac{F_{1}}{\nu_{1}}+\frac{F_{2}}{\nu_{2}}=\Delta F$ the com. bined chromatic convergence. If the combination is to be achromatic $\Delta F=0$, and hence $\frac{F_{1}}{\nu_{1}}+\frac{F_{2}}{\nu_{2}}=0$, or $F_{2}=-\frac{\nu_{2}}{\nu_{1}} F_{1}$.

Hence, we have $F_{1}=\frac{\nu_{1}}{\nu_{1}-v_{2}} F$ and $F_{2}=-\frac{\nu_{2}}{\nu_{1}-\nu_{2}} F$ which gives us in a moment the convergences of the component lenses required to produce an achromatic combination of any desired convergence.

For example, if we require an achromatic combination of $20 \mathrm{~cm}$. focal length of the following glasses:-

Chance's Hard Crown A. 605, $\mu_{D}=1.5175, v_{1}=60.5$.

Dense Flint A. 361, $\mu_{\mathrm{D}}=1.6214, \nu_{2}=36.1$.

Here the focal length of the combination is $20 \mathrm{~cm}$. and its convergence $\mathrm{F}=+5 \mathrm{D}$ and $\nu_{1}-\nu_{2}=24.4$. 
Hence, $F_{1}=\frac{60.5}{24.4} \times 5=+12.4 \mathrm{D}, \mathrm{C}_{1}=\frac{12.4}{.5175}=+$

$23.97 \mathrm{D}$, and $\mathrm{F}_{2}=-\frac{36.1}{24.4} \times 5=-7.4 \mathrm{D}, \mathrm{C}_{2}=-\frac{7.4}{.6214}$ $=-11.9 \mathrm{D}$. We therefore see that the combination must be made of $a+12.4 \mathrm{D}$ crown lens, having a total curvature of $23.97 \mathrm{D}$, and a - 7.4 D flint lens with a total curvature of - 11.9 D. The actual face curves will depend on whether or not the combination is to be cemented, and other aberrations are to be corrected.

It should be borne in mind that it is frequently desirable to under correct or over correct the combination by a certain amount to compensate for an eyepiece or other lens. This can be done immediately by putting $\frac{F_{1}}{v_{1}}+\frac{F_{2}}{v_{2}}=\Delta F=$ the required chromatic convergence, and we then have $F_{1}=\frac{\nu_{1}}{\nu_{1}-v_{2}} F+\frac{\nu_{1} \nu_{2}}{\nu_{1}-\nu_{2}} \Delta F$ where $\triangle F$ is the amount of over correction required.

In photographic work, as is well known, the efficiencies of the glass should be taken from the $D$ to the $G$ lines of the spectrum, instead of from the $\mathrm{C}$ to the $\mathrm{F}$ lines, as giren in the catalogues. These efficiencies can be calculated from the data given in the lists.

Chromatic Aberration of Lens Systems.-In the case of thin lenses the procedure given above enables us to completely correct a combination for chromatic aberration, with the exception of the small secondary spectrum which remains owing to the irrationality of the dispersion of the glass, and which can be reduced by the employment of suitable pairs of glasses. When, however, we come to lenses having a definite thickness, or to a combination of lenses which are separated by considerable distances, we find that chromatic aberration manifests itself in two ways, which are not simultaneously corrected as a rule. This will be understood in a moment if we consider the case of two thin lenses separated by a certain distance. If $f_{1}$ and $f_{2}$ are the focal lengths of the two lenses respectively, and $d$ the distance between them, we know that the "back focus" or distance from the second lens to the focal 
plane is equal to $\frac{f_{2}\left(f_{1}-d\right)}{f_{1}+f_{2}-d}$, while the equivalent focal length of the combination is $\frac{f_{1} f_{2}}{f_{1}+f_{2}-d}$. If we make the first amount the same for different colours, we shall then find that the images of a distant object will all lie in the same focal plane, in other words, there will be no chromatic difference of position, but it will be found that if this is done it will not as a rule make the quantity $\frac{f_{1} f_{2}}{f_{1}+f_{2}-d}$ the same for all colours, consequently the equivalent focal length will be different for the red and violet light, and the result will be that the image produced by one colour will be larger than that produced by the other. In this case we shall find that the image is perfectly colourless at the centre, but coloured at the margins, as is frequently the case; on the other hand, if we arrange that the equivalent focal length is the same for all colours it will be found, as a rule, that the images are formed in different focal planes. We are, therefore, led to distinguish between two principal kinds of chromatic aberration in lens systems, one the chromatic aberration of the position of the image, the other the chromatic aberration of the equivalent focal length or magnification. It would be impossible to go into this subject thoroughly without a fair amount of mathematical work, but it will be well to note that in the case of two thin lenses separated by a definite distance, which is a frequent combination for eye-pieces, we get the complete achromatism of the equivalent focal length by making both lenses of the same lind of glass and separating them by a distance equal to half the algebraic sum of their focal lengths, or $d=\frac{f_{1}+f_{2}}{2}$. This is the case in the Huyghens or negative eyepiece, which is, therefore, used whenever possible for microscope and telescope work. This eye-piece is not chromatically corrected for differences in the positions of the images, but this can be got orer in practice by slightly over correcting the object glass as indicated above.

Central Spherical Aberration.-The calculation of spherical aberration involves a certain amount of mathematical work, which 
I do not propose to give here. It will be sufficient if I mention that the calculation may be considerably simplified by the introduction of the curvature or dioptric system, and give the results obtained.

As is well known, the effect of central spherical aberration is to make the lens with a convergence or divergence stronger at the edges than at the centre. We-know that when light is refracted at a single spherical surface the ordinary formula is $\mu_{2} V-\mu_{1}$ $U=\left(\mu_{2}-\mu_{1}\right) R=F$ where $U$ and $T$ are the convergences of the light before and after refraction, $R$ the curvature of the surface, and $\mu_{1}$ and $\mu_{2}$ the indices of refraction of the first and second media respectively. When we come to consider the effect of spherical aberration we find that the formula becomes $\mu_{2} \mathrm{~V}-\mu_{1}$ $\mathrm{U}=\left(\mu_{2}+\mu_{1}\right) \mathrm{R}+\frac{\mu_{1}\left(\mu_{2}-\mu_{1}\right)}{\mu_{2}{ }^{2}}\left\{(\mathrm{R}-\mathrm{U})^{2}\left(\mu_{1} \mathrm{R}-\right.\right.$ $\left.\left(\mu_{2}+\mu_{1}\right) U\right)\left\{\frac{y^{2}}{2}\right.$ which equals $F+a \frac{\lambda y^{2}}{2}$ where $a=$ $\frac{\mu_{1}\left(\mu_{2}-\mu_{1}\right)}{\mu_{2}^{2}}\left\{(\mathrm{R}-\mathrm{U}) \cdot 2\left(\mu_{1} \cdot \mathrm{R}-\left(\mu_{2}+\mu_{1}\right) \mathrm{U}\right)\right\}$

This shows us, therefore, that the lens becomes stronger as we proceed from the centre to its edge, and that therease of its convergence is proportional to the square of the distance $y$ from the axis. The quantity $a \mathrm{I}$ have ventured to term the co-efficient of spherical aberration of the surface for the light considered.

If we consider the case of a thin lens of refractive index $\mu$ in air it is easily found that the co-efficient of spherical aberration $a$ for the lens is $\frac{\mu-1}{\mu^{2}}\left\{(\mathrm{R}-\mathrm{C})^{2}[\mathrm{R}-(\mu+1) \mathrm{U}]-(\mathrm{S}-\mathrm{V})^{2}\right.$ $[S-(\mu+1) V]\}$ where $R$ and $S$ are the curvatures of the front and back faces of the lens respectively, and $U$ and $V$ the convergences of the light before and after passing through the lens. If the incident light is parallel, as is the case in telescope and photographic lenses, $U=O$ and the formula then becomes $a=\frac{\mu-1}{\mu^{2}}$ $-\mathrm{R}^{3}-(\mathrm{S}-\mathrm{F})^{2}[\mathrm{~S}-(\mu+1) \mathrm{F}]$ 
This formula may be used to find the spherical aberration of any lens with very little difficulty, but it is possible to obtain the spherical aberration of any lens with hardly any calculation whatever by having recourse to curves plotted from the results of this equation. If, for example, we take a $+1 \mathrm{D}$ lens, we can calculate the co-efficient of spherical aberration for different values of the curvatures of one of the faces, say the back face, for glass of any particular refractive index, and on doing this we get the curve shown in fig. 10.

From this curve we see that if the back surface of the lens is plane or of no curvature the co-efficient of spherical aberration has a value of about 2.5 units, while with any other curve the

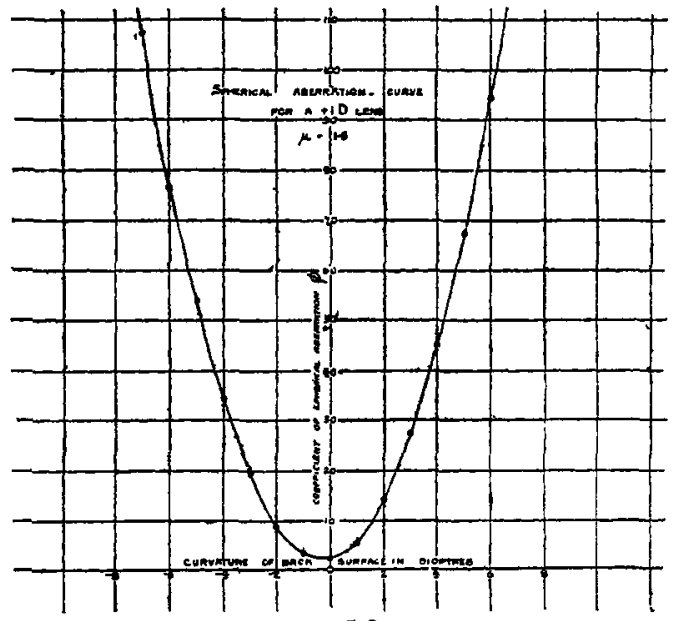

FIG. 10.

amount of splierical aberration is easily read off. If the lens has a conrergence of any other value we have merely to multiply the value of $a$ taken from the curve by the cube of the convergence in cliopters, and we then obtain the co-efficient for the lens for parallel incident light. Similar curves may be plotted for different values of the refractive index, and of the convergence of the incident light, and I have found them most valuable.

Aplanatic Points of a Sphere.-A principle which is extremely valuable in the correction of spherical aberration of microscope objectives was first introduced by Amici, and is mentioned here as it is not called attention to in most of our optical text books. 
Our formula above for the spherical aberration at any surface between the two media of any refractive indices shows that if $R=$ $\mathrm{U}$ or $\mu_{1} \mathrm{R}=\left(\mu_{1}-\mu_{2}\right) \mathrm{U}$ the co-efficient of spherical aberration is zero, in other words, the spherical aberration vanishes. In the first case the light is, of course, incident directly on the spherical surface, and no refraction is produced, consequently the refraction has no optical value, but in the second case where $U$ is equal to $\frac{\mu_{1}}{\mu_{1}+\mu_{2}} \mathrm{R}$ or $\mu=\frac{\mu_{1}+\mu_{2}}{\mu_{1}} r$ we have refraction without any spherical aberration, and it will be shown later without coma; this is illustrated in fig. 11, which shows a sphere of glass and two points $\mathbf{P}$ and $\mathbf{Q}$. If $r$ is the diameter of the sphere, and $\mu$ is its refractive index, then if $\mathrm{P}$ is at a distance from the

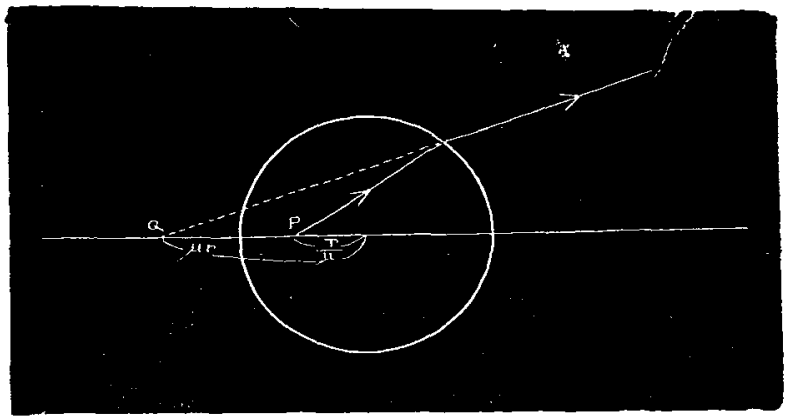

FIG. 11.

centre of the sphere equal to $\frac{r}{\mu}$ and $Q$ at a distance from the centre equal to $\mu r$, then the condition given above is satisfied, and every ray of light proceeding from $P$ will appear after refraction at the sphere to come from $Q$, however wide angled the pencil may be. It will be seen, therefore, that if instead of a complete sphere a hemisphere of glass is used, as in fig. 12, and the object is in front of the hemisphere at the correct distance, while between it and the hemisphere a liquid of the same refractive index as the glass is interposed, we shall have a perfect virtual image of the object at the point $Q$. This image may be further magnified by having a second lens behind the first, as shown, having the 
centre of curvature of its first face at the point $Q$, and its second face such that $Q$ is one of its aplanatic points, but it is not possible to form a real image in this way. In order to form a real image it is then necessary to use other lenses which are usually cemented over corrected combinations to compensate for the chromatic aberration of the hemisphere. This is, of course, the principle of the construction of immersion microscope objectives.

Coma.-If the errors of chromatic and central spherical aherra. Simare corrected, the image of an object on the axis is a perfect jmint. When, however, we have light emanating from a point. on one side of the axis, the light meets the lens olyiguely, and we then have a new series of errors of which the first and most inportant is coma. The effect of coma can he easily understood

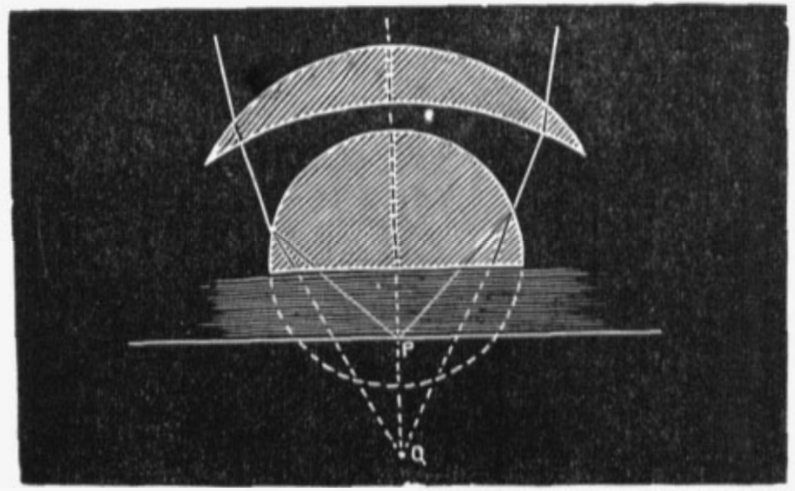

FIG. 12.

by reference to fig. 13. If we consider three rays from the object, one passing through the optical centre of the lens, while the other two pass through the upper and lower edges of it, it is obvious that in the case given the lower ray is refracted before the other, and consequently will intersect the central ray at a point closer to the lens than the other marginal ray does, the result will be that the light is not brought to a point focus, but to an unsymmetrical blur, from which the name coma is derived. Photographs of this defect were shown in my former paper. The effect of coma, however, depends. like that of spherical aberration, on the form of the lens, as will be seen on reference to figs. 13 and 14. As has been before said, the edge of the lens refracts like a prism, and 
the deviation produced by the edge is consequently dependent on the direction in which the light meet the surfaces. In fig. 13, the lens is double convex, and it is easily seen that not only is the light at the bottom refracted to a nearer point on account of its meeting the lens earlier, but it also suffers a greater deviation as it meets the surfaces more obliquely, while the deviation of the other side is actually reduced. On the other hand, if the lens is made convexo-plane, as in fig. 14, it will be seen that the effect of obliquity is to make the deviation of the bottom ray smation than that of the top, and hence to reduce the defect. In order to eliminate coma, therefore, our aim should be to construct the lens so that the effect of early refraction is compensated by diminished deviation, and this can be done with suitable lens combinations. It has been shown by Prof. Abbé, and more simply by Mr. Hockin, that the defect of coma is eliminated if what is called the sine corclition is satisfied, i.e., that the ratios of the sines of the angles

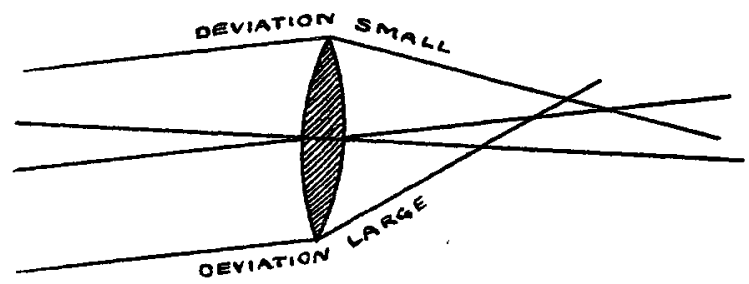

FIG. 13.

Which the conjugate rays malie with the axis are equal for points on the ans. Mr. Dennis Taylor has given a valuable method of correcting coma in the case of telescope objectives, and I have found that a combination of his formula with the curves for spherical aberration I have given above of considerable value. Coma is also eliminated in any system if the second of Von Seidel's conditions is complied with.

Radial Astigmatism.-This aberration is one which is easily understood with the aid of the wave or curvature theory, and is well known to Opticians in connection with tilted lenses. Figs. 15 and 16 show in plan and elevation what occurs when a plane wave of light is refracted at a tilted convergent lens, and it is seen inmediately that the effect of tilting is two-fold. In the first place the light has to pass through a greater thickness of glass, and in the second place, in the meridian perpendicular to the 
axis about which the lens is tilted, the lens acts as if it were of smaller aperture. Owing to these two causes, as is well known, the lens acts as a somewhat stronger spherical lens combined with a cylinder having its axis coincident with the axis of tilt.

If $\mathrm{F}$ is the convergence of the lens, $\mu$ its refractive index, and $\theta$ the angle through which it is turned, we find that the spherical convergence is increased by an amount equal to $\frac{\mathrm{F} \theta^{2}}{2 \mu}$, while the strength of the cylinder $=\mathrm{F} \theta_{2}$. The strength of this cylinder is the radial astigmatism, and it consequently follows that the radial astigmatism of any lens in diopters may be simply obtained by multiplying its convergence in diopters by the square of the angle of obliquity in radians. As this does not appear to be generally known, and may be of value in visual work, I give the

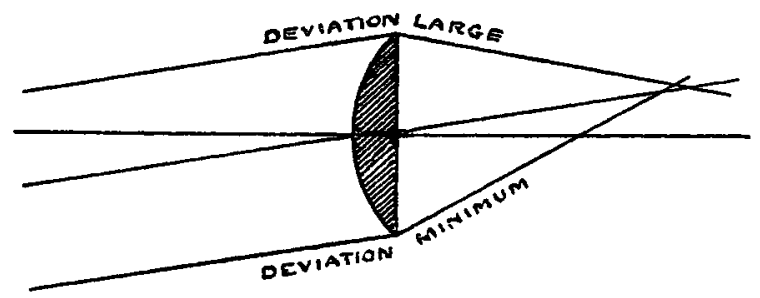

FIG. 14.

equivalent formula when $\theta$ is in degrees, we then find that the $\theta^{2}$

astigmatism in diopters equals $\frac{}{3280}$ multiplied by the convergence of the lens in diopters.

It will be noticed that since this astigmatism only depends on the convergence of the lens, and not in any way upon its refractive index or face curves, it is impossible in any way to correct or reduce the astigmatism of any combination of thin lenses in contact. It has frequently been assumed, therefore, that radial astigmatism is incapable of correction, but Von Seidel has shown that this is not the case for separated lenses or systems having considerable thicliness, and the astigmatism may be corrected by satisfying the third of his conditions.

Curvature of the Field. - If all the aberrations given up to the present are corrected, the result will be that a perfect image of 
an extended object can be produced, but, as is well known, it will lie on a curved surface instead of a plane, the amount of this curvature may be very considerable, as indicated in fig. 6 . When radial astigmatism is not corrected there are three surfaces, one containing all the primary focal lines, another all the secondary focal lines, while between them lies a third surface in which is contained all the circles of least confusion, which is the nearest approach we can get in this case to a perfect image. When astigmatism is completely corrected we have one surface only, corresponding to that of the circles of least confusion. The radii of curvature of the three surfaces are respectively $\frac{\mu}{\mu+1} t$,

FIG. 15 .

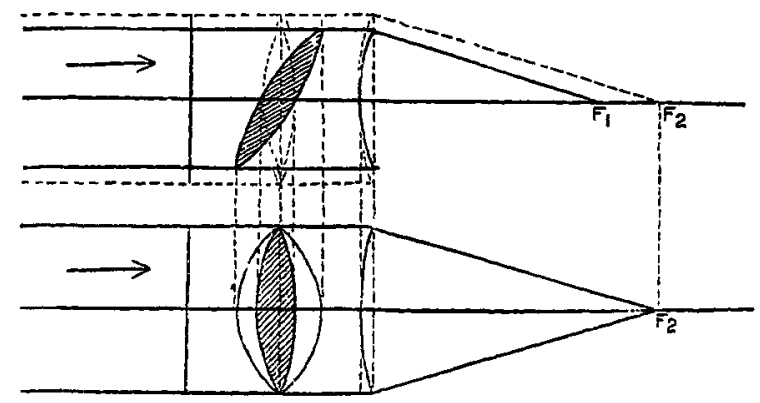

FIC. 16.

$$
\frac{\mu}{2 \mu+1} f, \text { and } \frac{\mu}{3 \mu+1} f
$$

If radial astigmatism has already been corrected, and we have a system of any number of thin lenses, we can make the image lie in one plane if the sum of the convergences of the lenses each divided by its respective refractive index is zero. For example, if we have two thin lenses either in contact or separated we shall have a flat field if $\frac{F_{1}}{\mu_{1}}+\frac{F_{2}}{\mu_{2}}=0$.

On referring to the section on chromatic aberration it will be seen that for two thin lenses in contact we have $F_{1}+F_{2}=F$ 
(convergence) and $\frac{F_{1}}{v_{1}}+\frac{F_{2}}{v_{2}}=O$ (achromatism), and we now have $\mathrm{F}_{1}+\frac{\mathrm{F}_{2}}{-}=\mathrm{O}$ (flat field).

$\mu_{1} \quad \mu_{2}$

These three conditions can only be satisfied at the same time if $\frac{v_{1}}{\mu_{1}}=\frac{\nu_{2}}{\mu_{2}}$ or if the efficiencies of the two glasses are proportional to their refractive indices. On reference to an ordinary table of optical glass it will be seen that as the refractive index of the glass increases the efficiency generally decreases, and until the anomalous Jena glasses were produced it was therefore impossible to produce such an achromatic combination of thin lenses in contact. Now, however, we have glasses in which the relation above given can be approximately fulfilled, and we are therefore able to produce what are termed new achromats having a flat field. I venture to think that it would be an additional assistance in lens design if optical glass manufacturers would give us, in addition to the information they already do, the values of the quotient $\frac{\nu}{\mu}$ for each kind of gilass.

There is, however, another method of correcting curvature which has been introduced by Mr. Dennis Taylor and Messrs. Busch, and again recently by Dr. Steinheil, in which the use of anomalous glasses is avoided. The condition for flat field is as given above $\frac{F_{1}}{\mu_{1}}+\frac{F_{2}}{\mu_{2}}=0$. If both lenses are of the same glass $F_{1}=-F_{2}$ and the lenses neutralise when in contact. On separating them, however, the combination now has a definite convergence with a flat field, and by extending this principle to three or more lenses, other aberrations may be corrected.

Distortion. - The question of distortion can be dismissed in a few words, as the defect is well known and its remedy in the case of photographic lenses also fairly well understood. It seems curious, however, that the general principle of its compensation does not seem to have been recognised until Lummer brought it forward in the year 1896, and it was independently found by 
myself and given in my lectures some four years ago. In order that the image should bear exact similarity to the object only one condition is required, that the chief rays proceeding from each point in the object to the diaphragm should, after refraction, emerge from a point on the axis, in other words, that the lens system should be aplanatic with respect to its entrance and exit pupils. When this is the case the image is formed precisely as in a pin hole camera, and there can subsequently be no distortion. It is therefore necessary to eliminate distortion to correct the spherical aberration, not only from positions of the object and image, but for the points at which the images of the diaphragm are formed. As is well known, and as is indicated by the formule and curves given above, spherical aberration is different for variations of the conjugate positions.

With this summary of the aberrations and indications as to thoir removal, I must bring this somewhat lengthy and imperfect paper to a conclusion. As I stated at the outset, my object las been rather to attempt to interest English Opticians in this injportant subject and to show that it is not so extremely intricate. In my opinion an immense assistance would be given to designers if tables and curves similar to those given in the paper were calculated, and I should like to see some members of the Optical Society saj, or others who took an interest in these things, get together and calculate out for us a set of tables, somewhat like Dr. Thompson's book of optical tables, but which only refers to elementary matters. I want to see spherical aberration, and other aberrations, set out, just as we see nautical almanacks, \&c., are got up; so that we could all refer to such a table, and get the spluerical aberration of a lens, and compensate it without very much difficulty. It can be done with concerted and co-operative action. Mr. Salt asked for co-operative action in certain directions of the optical trade. May I ask that we should try and co-operate in pushing forward some of the higher branches of the optical industry. I think we should try to raise our status in that direction as well as in every other. (Cheers.)

The Chairmas : You have heard Dr. Drysdale's paper, and he has kindly invited you to comment on it; therefore, I should be cxceedingly glad if anybody would do so.

Mr. DIxIE: May I have the privilege of proposing a very cordial vote of thanks to Dr. Drysdale for the most interesting 
and able paper which he has read us this evening? The whole papes bristles with interesting points, and there are a great number of questions which I should like to ask Dr. Drysdale, but really one does not know where to begin. The most interesting point, and that most present in my mind now, is a question that ayose on the last diagram that was thrown on the screen. I assume that that curve was calculated for parallel light. What would be the effect if other sources of light were used? That is one thing. Another thing, perhaps, might be worth mentioning : On behalf of working Opticians, in a humble ray, I desire to express my great sense of gratification that a scientific gentleman like Dr. Drysdale has bent his mind to the study of expressing mathematical formulæ based on the dioptric system instead of the focal length system, or whatever the old system may be called. I am convinced mJself, without professing to any special knowledge on the point-it seems to me much more rational altogether to base your formulæ in your calculations on the first quality of the lens; and I an pretty well convinced that just as we have proved it of great advantage in our spectacle work-we have been practically driven to it-so I have no doubt that it will be ir the construction of the more intricate instruments. I have no doubt it is the duty of us practical men to give a certain amount of thought to this ; but it is more the duty, I think, of gentlemen in the position of Dr. Drysdale, who, after all, have nothing to do but think, or very little else-(laughter), - to hammer out some definite system for us. With regard to the dioptric system,-I always like to be practical, and if you would not mind I would like to show Jou what I mean on the blackboard. Supposing we take the very simplest formula, what you begin to teach your elementary wooden-heads, and which is in almost the very first lecture you give them-the law of conjugate foci. I could show rou how very simple it would be to teach that in simply adopting liopters. I have a lens here which we will say is +10 diopters. We are in the habit of writing it like that-all spectacle men know exactly what that means,- - but if you are talking to a class you have to talk about the focal lengths. Very well, now, you take your point here, $\frac{1}{4} \mathrm{~m}$. from the centre of the lens. Then we know that in order to bring that light to where we see it, all the diop.ers of our lens which I write thus are used. What have we left? Four from $10=6 ;+6$ left. Therefore, if we want to find the 
focus which is con juyate to that point, this is like teaching that six and four are 10. That entirety explains the matter for a thin lens-it entirely explains the principle of conjugate foci. of cousse, it nay be extended-you may take the thickness into accourit, or a simple lens system into account,-add the distance hetween the principal planes, and so on. From that you could develop a great deal of the more complicated lens system. Again I thank Dr. Drysiale very much for the lecture he has given us, and I ask you to allow me to propose a very cordial vote of thanks. (Cheers.)

Mr. SALT : Mr. Chairman and gentlemen, I do not intend for one moment to criticise Dr. Drysdale's paper, nor to offer any counter or other suggestions as to the question of these lenses; but I feel proud in being able to second Mr. Dixey's vote of thanks

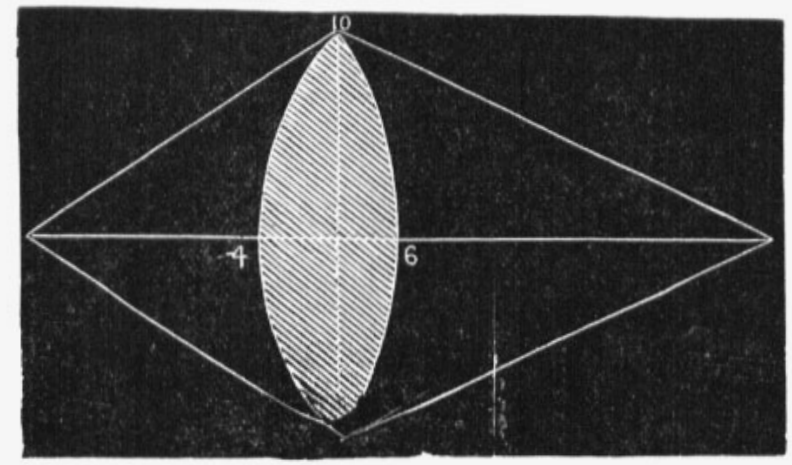

to Dr. Drysdale, who has always been so very kind in giving us the benefit of his "thinking" powers, which Mr. Dixey spoke of ; and I do sincerely join Mr. Dixey in offering the lecturer our thanks for giving us his paper to-night. (Cheers.)

Dr. Mullineux Wamster: I had not the slightest indication of the line Dr. Drysdale was going to take in this paper, nor did I know at all what he was going to say. The first part of his paper was introductory, and was a very strong plea for an 0ptoTechnical Institute, if for nothing else. (Hear, hear.) In regard to Dr. Drysdale's remarks on that subject, we do know that modern methods of manufacture have gone ahead at a tremendous rate. At this late hour one cannot pretend to go over all the points Dr. Drysdale has raised. In regard to what he said about 
computers, a British astronomer found the planet Neptune, a task quite equal to any optical problem you can set. British computers have arrived at a very perfect linowledge of the planets; but we have not yet been able to convince them that optical problems are worthy of their attention. Therefore I say we have the computers in the country; and if the optical trade can only convince them that these computations will be for the advancement of optical linowledge, so much the better. But far more important than questions of computation or dead level calculation is the question of the development of the photographic lens-a question which I venture to thinls Dr. Drysdale's paper has advanced very considerably. Those early methods of Grubb are old and well known-well known in one sense, but I doubt whether they are known to most teachers of optics, simple though they are. And the method of working in curvatures instead of in focal distances and linear distances, I should say is almost unknown ground, outside the optical trade. Personally, I have taught to all my elementary optical students during 15 or 16 years, working in curvatures instead of linear distances; but until I went to the Northampton Institute my students were never students in connection with the optical trade, and teaching the general science of optics as pure science, one never carried it far enough. But the ordinary student could quite as easily work with the curvatures (at least, that has been my experience), whereas if it is left until the time when the student becomes the practical Optician it is too overwhelming a subject for him to talie it up at all. I think you will agree with me that we are very fortunate in having at the Northampton Institute one so competent to teach the science of optics as Dr. Drysdale. (Cheers.) I should like to say, if I may do so without any suspicion of boasting, that the first task of leading the optical student forth on scientific lines really fell, at the Northampton Institute, into Dr. Drysdale's hands; and I consider that was a very fortunate thing indeed. One of the greatest advantages in Dr. Drysdale's methods in regard to this matter, is the saving in the computation time. You may eventually have to make a final calculation, because the method is obviously only an approximation, but it is so very nearly accurate as to be sufficient for practical purposes. I may say for 15 or 16 years I have been dealing with these matters. You have only to lonk at the formulæ, commencing with 1 over this, that 
and the other-1 over linear distance is curvature; whatever may appenr in the formula, or whatever the symbol, if only linear distance is the denominator it represents a curvature in the system. Curvatures are the things for Opticians, and linear distances are only to be used by astronomers. As regards tables being calculated by the Society, we have heard of that being done by other societies, in other directions. I might venture to suggest that even more useful than tables would be outlines of curves, such as we saw on the screen. Cut out the template block, lay it down on your drawing paper on these outlines, and you get rour approximate solution. It would take you a considerable amount of labour, of dead calculation, either by yourself or a deputy computer, in order to get such approximate results. Outlines of curres, I venture to think, world be far more useful to the optical trade than the diagrams relating to dynamo-electrical machinery, published in Germany, which are for the use of the electrical tıadc. (Cheers.)

The Chatrman : The motion before you is that a very cordial vote of thanks be accorded to Dr. Drysdale for his paper. Will you kindly signify your approval, gentlemen, in the usual manner?

The vote of thanks was carried by acclamation.

Dr. DRYSDaLE: Gentlemen, I have to thank you very cordially for the kind way in which you have expressed your approval of the very slender information I gave you in ny paper. It was only a very small portion of what I would have liked to give you in the time. At any rate, I hope the few remarks made may lead rou to take more interest in the subject, if you have an opportunity of secing the lecture in print. I do not think I have very much to reply to in the discussion. I thanls those who have spoken for the kind remarks made about these methods. As far as Mr. Dixey's observations went, one point he raised has some interestWhether the curves would be suitable for different convergences. I do not propose them to be used where time permits of a calculation; but it will be found in practice, if you are going to use a telescope combination, one of the lenses of the combination does have parallel light passing through it. It would be very easy for you to deal with such a case. If Mr. Dixey wishes it I can give him further information on that point. I should like, perhaps, to take a little exception to his remark that we have nothing to do 
at the Northampton Institute but think out such things. I want to say that we have a very little time to think out such things; but perhaps that is not a very happy way of putting it, as we cannot get at these conclusions without thinking. The portion of our time that we can devote to optics is very slender, for the number of students we have in the optical department is, unfortunately, but a small porportion of the whole, and naturally most of our attention has to be given to the greater number. As thing develop we hope that may not be the case. (Hear, hear.) I do not think I need say anything about the point raised bv Dr. Walmsley. I do not forget that we have excellent computers in the country; but the main difficulty is that the man who does the computation in optics must be a practical man also You must have a man with a practical idea of what he wants to get before he will be useful to practical Opticians. Instead of a person who computes in a mechanical manner, when he has a formula adjusted, we want the combination of a scientific man who is able to use these calculations in a practical manner. We have got to do that if we are going to keep abreast of the times. As regards the curves, my suggestion is that the Optical Society should prepare a table of these things-that they should also give us the curves, which are of more value than the tables, but are not quite accurate enough for final calculation. At any rate, curves are of such enormous assistance that a collection of curves should follow the tables. I thank you very cordially for all the kind things you have said. (Cheers.) 